\title{
QUALITY OF LIFE AND ITS RELATED FACTORS AMONG PARTICIPANTS IN SHAHREKORD - IRAN COHORT STUDY
}

\author{
${ }^{1}$ Shahrekord University of Medical Sciences, Shahrekord, Iran \\ ${ }^{2}$ Social Medicine Department, Shahrekord University of Medical Sciences, Shahrekord, Iran \\ ${ }^{3}$ Social Determinants of Health Research Center, School of Allied Medical Sciences, Shahrekord University \\ of Medical Sciences, Shahrekord, Iran \\ ${ }^{4}$ Psychology Department, Shahrekord University of Medical Sciences, Shahrekord, Iran
}

\begin{abstract}
PURPOSE. This study aimed to determine quality of life and its related factors among participants in Shahrekord Cohort Study in southwest of Iran.

DESIGN/METHODOLOGY/APPROACH. The present study was a descriptive-analytical cross-sectional study approved by Shahrekord University of Medical Sciences. One thousand participants in the Shahrekord cohort study were selected through a convenience sampling method, and entered the study based on the inclusion criteria. Participation in the study was completely voluntary, and a written consent form was obtained from each participant. Data gathering tools were a demographic questionnaire and the short version of the World Health Organization Quality of Life (WHOQOL-BREF) questionnaire. Data were collected through face-toface interviews by trained individuals. Collected data were then analyzed using SPSS v24.

FINDINGS. No significant relationship was observed between the physical health and also the environmental health and participants' sex. However, in other dimensions of quality of life including psychological health, the social relationship health, general perception of health, and the overall quality of life score, male participants reported a better quality of life $(\mathrm{p}<0.05)$. No significant relationship was observed between the physical health, the psychological health, and the general perception of health with participants' levels of education. However, there was a significant relationship between the social relationship health, the environmental health, and the overall score of quality of life with participants' levels of education $(p<0.05)$. In other words, higher level of education improved the social relationship health, the environmental health, and the overall score of quality of life. Being married in men showed a positive relationship with the social health, the general perception of health, the psychological health, and the overall quality of life score $(\mathrm{p}<0.05)$. However, the physical health and the environmental health had no relationship with marital status in men. Being married in women only improved the social relationship health, and it was not associated with other dimensions measured by the WHOQOL-BREF questionnaire. Smoking, addiction, alcohol consumption, and cell phone use were not associated with any of the dimensions measured in the questionnaire. Diabetes caused a decline in the environmental health as well as the general perception of health $(\mathrm{p}<0.05)$. Cardiovascular disease also caused a decline in the psychological health, the social relationship health, and the general perception of health $(\mathrm{p}<0.05)$.

ORIGINALITY/VALUE. The results of the present study showed that female, illiterate and unmarried participants, and those with diabetes and cardiovascular diseases reported a poorer quality of life in some dimensions compared to other participants; using the cell phone, smoking, alcohol consumption, and addiction had no relationship with the quality of life.
\end{abstract}

Keywords: quality of life, cohort study, chronic diseases, addiction, alcohol consumption 


\section{INTRODUCTION}

Chronic diseases are defined as diseases whose effects are persistent and/or long-lasting (1). The term chronic is usually used when the disease lasts more than three months. Chronic diseases often have a progressive and changeable course, and they may include frequent recurrences along with periods of recovery. If left untreated, these diseases can result in other chronic diseases (2). Nowadays, chronic diseases are addressed as a challenge to global health (3). In a report in 2010 provided by the World Health Organization on the challenge of chronic diseases, it was stated that non communicable diseases such as cardiovascular disease, diabetes, cancers, and respiratory diseases account for approximately twothirds of all deaths worldwide (4). In the United States, chronic diseases are the leading cause of poor health, disability and death, and they impose high costs on the healthcare system (5). About half of the adults $(50.9 \%)$ in the United States have at least one type of chronic diseases, and $26 \%$ have two or more chronic diseases simultaneously (6). In 2011, it was reported that $13.1 \%$ of the US population had a disability, of which $46.3 \%$ aged over 75 years (7). In Iran, the general statistics on chronic diseases, and a list of 12 major causes of death over the past 10 years and their trend of changes (increase or decrease) are presented in the latest global burden of disease data; and cardiovascular diseases with $27 \%$ increase, stroke with 19\% increase, Alzheimer's disease with $103 \%$ increase, road injuries with $31 \%$ decrease, hypertensive heart disease with $55 \%$ increase, diabetes with $76 \%$ increase, chronic obstructive pulmonary disease with $40 \%$ increase, chronic kidney diseases with 56\% increase, neonatal disorders with $60 \%$ decrease, respiratory infectious diseases with $0.6 \%$ increase, congenital defects with $54 \%$ decrease are addressed as the most important causes of death in Iran (8).

The complications caused by chronic diseases result in pathophysiological changes throughout the body that impose many problems on the patients and the healthcare system. Chronic diseases can have negative impacts on physical performance, psychological status, personal, family and social relationships, and in general, on a person's quality of life (9). Chronic diseases, due to their incurable nature as well as their long-term complications, reduce a person's ability to adapt, and are associated with some psychological complications (2). The limitations caused by chronic diseases, the use of oral or injectable drugs, and the long-term complications of the diseases have adverse effects on health outcomes, such as quality of life (10). Studies in diabetic patients showed that the problems imposed by diabetes such the necessity to follow a specific diet, activity restrictions, invasive blood glucose monitoring, daily insulin injection, chronic psychological conditions, hospitalization, and reduced median life expectancy in these patients affect their mental health and quality of life (11). In addition, studies showed that aging, low income, limited physical activity, insulin use, high body mass index, smoking, being female, poor sleep quality, and depression are among the factors associated with poor quality of life in patients with type 2 diabetes (12). In recent years, quality of life has been recognized as part of health, and it is considered in the evaluation of healthcare programs. Evaluating the quality of life help the assessment of health status, the effectiveness of medical and health interventions, disease assessment, cost-effectiveness of health care policies, and planning. Therefore, a better understanding of the quality of life and the factors affecting it among people with chronic diseases is considered essential to design and guide interventional strategies in order to alleviate the complications of these diseases. Hence, the present study was designed with the aim of determining the quality of life and its effective factors among participants in Shahrekord-Iran cohort study.

\section{METHODS}

The present study was a descriptive-analytical cross-sectional study approved by Shahrekord University of Medical Sciences-IRAN in 2019 (Ethical Code IR.SKUMS.REC.1397.270). One thousand participants in the Shahrekord cohort study, who had signed the consent form, were selected through convenience sampling methods, and their data on demographic variables and quality of life were collected and analyzed. The study population included participants in the Shahrekord cohort study in 2018. Participation in the study was completely voluntary, and after explaining the methods of the study, the written consent form was obtained from each participant. Data gathering tools were a demographic questionnaire and the short version of the World Health Organization Quality of Life (WHOQOLBREF) questionnaire. The WHOQOL-BREF questionnaire consists of 26 items, and its validity has been confirmed for the Iranian population. This questionnaire evaluates the quality of life through four dimensions of physical health (7 items, total scores of 7-35), psychological health (6 items, total scores of 6-30), social relationship health (3 items, total scores of 3-15) and environment health (8 items, total scores of 8-40), the general perception of health ( 1 item, total scores of 1-5), and an overall quality of life (1 item, total scores of 1-5). Each item includes 5 options, 
and is scored from 1 to 5 . In this questionnaire, some of the items are scored in a reverse manner. Data were collected through face-to-face interviews, and by trained individuals. Demographic variables included smoking status, alcohol consumption, opium addiction, medical history, cell phone use, body mass index, living conditions, marriage condition, age, sex, and educational status.

Inclusion and Exclusion Criteria. In this study the inclusion criteria were: age between 35 and 70 years and living in Chaharmahal and Bakhtiari province during the last 5 years. The Exclusion Criteria were the presence of a known mental disorder, the occurrence of brain injury complications, and failure to respond to the items of the questionnaire.

The Validity and Reliability of Data Gathering Tools. In this study, the short version of the World Health Organization Quality of Life (WHOQOLBREF) questionnaire was used, which it has an appropriate validity and reliability (Cronbach's alpha of 0.922) (13). Collected data were analyzed using SPSS v24. For descriptive analysis mean \pm Standard deviation and frequency used for quantitative and qualitative data respectively. Also according to the type of variables chi-square, independent t-test, and one-way analysis of variance were used for comparison between groups. P-value of less than 0.05 was considered significant.

\section{RESULTS}

The results of the study population's demographic variables are presented in Table 1 and 2. Among participants $48.6 \%(n=486)$ were male, $95.5 \%$ were married, $30 \%$ were illiterate, $44.5 \%$ had elementary or high school education, and $25.5 \%$ had academic education. $14 \%$ were smoker, $16.2 \%$ were addicted, and $15.3 \%$ consumed alcohol. $4 \%$ had cardiovascular diseases and $6 \%$ suffered from diabetes.

Based on the results, no significant relationship was observed between the physical health and the environmental health with participants' sex ( $p>0.05)$. However, there was a statistically significant difference between male and female participants regarding their score in psychological health, social relationship health, the general perception of health, and the overall score of quality of life (Table 2). Male participants reported better quality of life in these dimensions.

According to the results of ANOVA test, educational level and physical health had not any significant statistical relationship, although in other dimensions of quality of life higher scores were seen in higher educational levels. Those with secondary school education had lower psychological health score than those with academic education ( $\mathrm{p}$-value $=.016$ ). Regarding the social relationship health those with elementary education and secondary school education

Table 1. The results of demographic variables among participants in Shahrekord cohort study.

\begin{tabular}{|l|c|c|c|c|c|}
\hline \multicolumn{1}{|c|}{ Variable } & Frequency & Min & Max & Mean & SD \\
\hline Age & 1000 & 35 & 70 & 48.99 & 9.149 \\
\hline Weight & 1000 & 36 & 125 & 73.157 & 12.964 \\
\hline BMI & 1000 & 15.79 & 51.72 & 27.232 & 4.484 \\
\hline Number of rooms & 979 & 0 & 9 & 2.17 & 0.817 \\
\hline Number of household members & 979 & 1 & 13 & 4.11 & 1.385 \\
\hline Residential space of the house $\left(\mathrm{m}^{2}\right)$ & 979 & 32 & 400 & 134.4 & 48.55 \\
\hline Years of using cell phone & 891 & 1 & 25 & 9.97 & 5.123 \\
\hline Diastolic blood pressure & 1000 & 50 & 110 & 75.97 & 11.068 \\
\hline Systolic blood pressure & 1000 & 78 & 202 & 115.97 & 17.862 \\
\hline
\end{tabular}

Table 2. The relationship between sexes, the dimensions of quality of life

\begin{tabular}{|l|c|c|c|}
\hline \multirow{2}{*}{\multicolumn{1}{c|}{ Dimensions }} & \multicolumn{2}{c|}{ Mean \pm SD } & \multirow{2}{*}{ p-value } \\
\cline { 2 - 4 } & Male participants & Female participants & .20 \\
\hline Physical health & $58.41 \pm 13.7$ & $57.30 \pm 13.7$ & .11 \\
\hline Environmental health & $65.73 \pm 15.1$ & $64.18 \pm 15.8$ & $<0.001^{*}$ \\
\hline Psychological health & $68.45 \pm 16.7$ & $63.28 \pm 16.5$ & $.008^{*}$ \\
\hline Social relationship health & $67.64 \pm 19.4$ & $64.34 \pm 19.9$ & $.01^{*}$ \\
\hline General perception of health & $68.26 \pm 19.4$ & $65.19 \pm 17.9$ & $.001^{*}$ \\
\hline The overall quality of life & $64.80 \pm 12.9$ & $62.22 \pm 12.6$ & \multicolumn{2}{c|}{} \\
\hline
\end{tabular}

* p-value of less than 0.05 was considered significant 
had lower scores than those with academic education ( $\mathrm{p}$-value $=.018$ and .001 , respectively).

Regarding the environmental health, there was no significant difference between participants with primary education and participants in other groups ( $p>0.05$ ), except with those with postgraduate and doctorate education ( $p$-value $=.013$ ). It also was shown that illiterate people had statistically significant lower scores than those with bachelor and post-graduate education ( $p$-value $=.002)$, and those with secondary school education had lower scores than all other higher educational groups ( $\mathrm{p}$-value $=.003)$. The mean of social relationship health score was significantly lower in participants with primary school education in compare to those with post-graduate education ( $\mathrm{p}$-value= .018) and those with secondary school education had lower social health scores than those with academic education ( $\mathrm{p}$-value $=.001$ ). The mean scores of general perception of health were lower in illiterate, primary school, and secondary school education in compare to those with academic education ( $p$-value $=.004, .018$, and .016, respectively). The overall quality of life scores were statistically significant lower in illiterate $(p$-value $=.009)$ and primary school $(p$-value $=.015)$ groups in compare with academic education group, and participants with secondary school education had lower overall score in compare with all other higher educational groups $(\mathrm{p}$-value $=.001)$.

In the current study there were statistically significant differences between single and married participants in some dimensions of quality of life. Married persons had higher psychological health $(p$-value $=.001)$, social relationship health ( $p$-value
$<.001)$, general perception of health $(p-v a l u e=.032)$, and the overall quality of life scores $(p$-value $=.004)$, while the physical health and environmental health scores did not show any significant differences between these two groups ( $p$-value $=.97$ and .73 , respectively). However, the relationship between quality of life and marriage was different between men and women and the positive effect of marriage on quality of life scores were more significant among male participants. In men being married showed a significant positive effect on their scores in Psychological health, Social relationship health, General perception of health, and the Overall quality of life, but in women married participants had statistically significant higher scores only in Social relationship health (Table 3).

In the current study smoking, addiction, alcohol consumption, and cell phone use were not associated with any of the dimensions measured in the questionnaire $(p>0.05)$. Diabetes was significantly associated with the overall perception of health and diabetic patients had lower scores in that dimension compare with non-diabetic participants. ( $p$-value= .016). No statistically significant difference was observed regarding other factors $(\mathrm{p}>0.05)$. Also, cardiovascular diseases were significantly associated with the lower psychological health $(\mathrm{p}=.002)$, social relationship health (.009), and the general perception of health scores (.001).

\section{DISCUSSION}

The differences observed in the scores of quality of life with respect to demographic variables showed

Table 3. The relationship between marital status in male and female participants with the dimensions and the overall quality of life scores.

\begin{tabular}{|l|c|c|c|c|}
\hline \multirow{2}{*}{\multicolumn{1}{|c|}{ Dimensions }} & \multirow{2}{*}{ Mean SD } & \multirow{2}{*}{ p-value } \\
\cline { 2 - 5 } & & $\begin{array}{c}\text { married } \\
\text { participants }\end{array}$ & $\begin{array}{c}\text { single } \\
\text { participants }\end{array}$ & .45 \\
\hline \multirow{2}{*}{ Physical health } & Male & $58.35 \pm 13.7$ & $62.05 \pm 16.3$ & .83 \\
\hline \multirow{2}{*}{ Environmental health } & Female & $57.33 \pm 13.7$ & $56.85 \pm 13.8$ & .69 \\
\hline \multirow{2}{*}{ Psychological health } & Male & $65.78 \pm 15.1$ & $63.67 \pm 14.3$ & .97 \\
\cline { 2 - 5 } & Female & $64.14 \pm 16.0$ & $64.27 \pm 13.8$ & $<0.001^{*}$ \\
\hline \multirow{2}{*}{ Social relationship health } & Male & $68.81 \pm 16.23$ & $47.39 \pm 31.4$ & .19 \\
\hline \multirow{2}{*}{ General perception of health } & Female & $63.45 \pm 16.2$ & $59.90 \pm 20.5$ & $<0.001^{*}$ \\
\cline { 2 - 5 } & Male & $68.13 \pm 18.8$ & $38.54 \pm 28.5$ & $<0.001^{*}$ \\
\cline { 2 - 5 } & Female & $66.00 \pm 19.0$ & $43.01 \pm 19.8$ & $.014^{*}$ \\
\hline \multirow{2}{*}{ The overall quality of life } & Male & $68.54 \pm 19.0$ & $51.56 \pm 34.9$ & .42 \\
\cline { 2 - 5 } & Female & $65.38 \pm 17.8$ & $62.83 \pm 18.5$ & $.043^{*}$ \\
\hline
\end{tabular}

* p-value of less than 0.05 was considered significant. 
that female, illiterate and unmarried participants had lower scores in some dimensions of quality of life compared to male, well-educated, and married ones, respectively. These findings have been well demonstrated in previous studies (14-17). The results of the present study showed that smoking did not significantly change the participants' quality of life. The results of one study conducted by Castro et al. showed that smokers with severe addiction scored lower, and those with moderate and mild addiction did not differ in terms of the scores obtained compared to normal individuals (18). These results indicate that in order to evaluate the effect of smoking, the study population should be well classified in terms of smoking, and our study was limited in this regard. The contradictory results between these two studies may be due to this limitation. Regarding alcohol consumption and addiction, the results of the present study did not show any significant differences between participants without a history of alcohol consumption and addiction. The results of one study conducted by Moreira et al. showed that the participants in control group, who did not have a history of alcohol consumption and addiction, had higher scores through all dimensions of the questionnaire compared to the participants involved with these substances (19). In the present study, the number of participants who consumed alcohol was 150, and the number of participants with addiction was 159 . This number of samples seems to be a small community to evaluate the role of the mentioned factors in quality of life and the contradictory results between these two studies can be due to the bias and the fact that because of the cultural issues in the country, alcohol consumption and addiction are considered abominable in and there is a possibility of under reporting among participants. Regarding the use of cell phone, the results of the present study showed that the use or non-use of cell phones makes no difference regarding the participants' quality of life. In this regard, Ghasemi et al. showed that the balanced use of technology can improve the quality of life in individuals, but not using or using more than two hours a day make no major differences regarding the quality of life (20). The results of the present study showed that diabetes caused a decline in the the general perception of health. In this regard, Jain et al. showed that individuals with type 2 diabetes scored lower compared to the group of healthy people regarding the physical health, the psychological health, the social relationship health and the environmental health (21). The results of the mentioned study are somewhat in line with the results of the present study, and the reason for the difference in some parts may be due to the existing cultural differences between the studied communities. Regarding the relationship between cardiovascular diseases and quality of life, the results of the present study showed that cardiovascular diseases caused a decline in the psychological health, the social relationship health, and the general perception of health. In this regard, Juenger et al. showed that cardiovascular diseases reduce the scores of quality of life through all dimensions of the WHOQOL-BREF questionnaire (22). The results of the mentioned study are in line with the results of the present study and in our study CVDs affected different dimensions of quality of life negatively.

\section{CONCLUSIONS}

The results of the present study, which was conducted among participants in Shahrekord cohort study, showed that female, illiterate and unmarried participants, and those with diabetes and cardiovascular diseases reported a poorer quality of life compared to other participants. Cell phone use, smoking, alcohol consumption, and addiction had no relationship with the dimensions of quality of life.

\section{REFERENCES}

1. Bauer UE, Briss PA, Goodman RA, et al. Prevention of chronic disease in the 21st century: elimination of the leading preventable causes of premature death and disability in the USA. Lancet. 2014;384(9937):45-52.

2. Ware Jr JE, Gandek B, Allison J. The validity of disease-specific quality of life attributions among adults with multiple chronic conditions. Int J Stat Med Res. 2016;5(1):17.

3. Murray CJ, Vos T, Lozano R, et al. Disabilityadjusted life years (DALYs) for 291 diseases and injuries in 21 regions, 1990-2010: a systematic analysis for the Global Burden of Disease Study 2010. Lancet. 2012;380(9859):2197-223

4. World Health Organization. (2014)? Global status report on noncommunicable diseases 2014: World Health Organization; 2014.

5. Schenck A, Andrulis D, Bartram J, et al. America's Health Rankings: A Call to Action for Individuals $\&$ Their Communities. Minnetonka: United Health Foundation. 2014.

6. Boersma P, Black LI, Ward BW. Peer Reviewed: Prevalence of Multiple Chronic Conditions Among US Adults, 2018. Prev Chronic Dis. 2020;17: E106.

7. Adams PF, Kirzinger WK, Martinez ME. Summary health statistics for the US population: National Health Interview Survey, 2011. Vital Health Stat 10. 2012(255):1-110.

8. 2018 DDRITDDRI. Outline the results of the Global burden of disease study in 2017 Iran. Available from: https://opizocom/TJLBDR2018. 
9. Scott KM, Lim C, Al-Hamzawi A, et al. Association of mental disorders with subsequent chronic physical conditions: world mental health surveys from 17 countries. JAMA Psychiatry. 2016;73(2):150-8.

10. Mielck A, Vogelmann M, Leidl R. Healthrelated quality of life and socioeconomic status: inequalities among adults with a chronic disease. Health Qual Life Outcomes. 2014;12(1):1-10.

11. Lustman PJ, Griffith LS, Clouse RE, et al. Psychiatric illness in diabetes mellitus: Relationship to symptoms and glucose control. J Nerv Ment Dis. 1986;174(12):736-42.

12. Lou P, Qin Y, Zhang P, et al. Association of sleep quality and quality of life in type 2 diabetes mellitus: a cross-sectional study in China. Diabetes Res Clin Pract. 2015;107(1):69-76.

13. Nejat S, Montazeri A, Holakouie Naieni K, et al. The World Health Organization quality of Life (WHOQOL-BREF) questionnaire: Translation and validation study of the Iranian version. sjsph. 2006; 4 (4) :1-12. Available at: http://sjsph.tums. ac.ir/article-1-187-en.html

14. Kenny C. Does development make you happy? Subjective wellbeing and economic growth in developing countries. Social Indicators Research. 2005;73(2):199-219.

15. Ohaeri JU, Awadalla AW, Gado OM. Subjective quality of life in a nationwide sample of Kuwaiti subjects using the short version of the WHO quality of life instrument. Soc Psychiatry Psychiatr Epidemiol. 2009;44(8):693-701.

16. Oswald $\mathrm{AJ}, \mathrm{Wu} \mathrm{S}$. Objective confirmation of subjective measures of human well-being: Evidence from the USA. Science. 2010;327(5965):576-9.

17. Skevington SM, Lotfy M, O'Connell KA. The World Health Organization's WHOQOL-BREF quality of life assessment: psychometric properties and results of the international field trial. A report from the WHOQOL group. Qual Life Res. 2004;13(2):299-310.

18. Castro MdG, Oliveira MdS, Miguel AC, et al. WHOQOL-BREF psychometric properties in a sample of smokers. Braz J Psychiatry. 2007;29(3):254-7.

19. Moreira TdC, Figueiró LR, Fernandes S, et a. Quality of life of users of psychoactive substances, relatives, and non-users assessed using the WHOQOL-BREF. Cien Saude Colet. 2013;18:195362.

20. Ghasemi SR, Rajabi Gilan N, Reshadat S, et al. Investigating Health-Related Quality of Life and the Use of Media Technologies in Adolescents. J Mazandaran Univ Med Sci. 2019;29(171):81-91.

21. Jain V, Shivkumar S, Gupta O. Health-related quality of life (hr-qol) in patients with type 2 diabetes mellitus. N Am J Med Sci. 2014;6(2):96.

22. Juenger J, Schellberg D, Kraemer S, et al. Health related quality of life in patients with congestive heart failure: comparison with other chronic diseases and relation to functional variables. Heart. 2002;87(3):235-41.

Received: 14.05.2021

Accepted for publication: 29.06.2021

\section{Address for correspondence:}

Ali Hasanpour Dehkordi

Social Determinants of Health Research Center,

School of Allied Medical Sciences,

Shahrekord University of Medical Sciences,

Shahrekord, Iran

e-mail: alihassanpourdehkordi@gmail.com 